www.nature.com/jhg

\title{
Association of variations in the FTO, SCG3 and MTMR9 genes with metabolic syndrome in a Japanese population
}

\author{
Kikuko Hotta ${ }^{1}$, Takuya Kitamoto ${ }^{1}$, Aya Kitamoto ${ }^{1}$, Seiho Mizusawa ${ }^{2}$, Tomoaki Matsuo ${ }^{3}$, Yoshio Nakata ${ }^{3}$, \\ Seika Kamohara ${ }^{4}$, Nobuyuki Miyatake ${ }^{5}$, Kazuaki Kotani ${ }^{6}$, Ryoya Komatsu ${ }^{7}$, Naoto Itoh $^{8}$, Ikuo Mineo ${ }^{9}$, \\ Jun Wada ${ }^{10}$, Masato Yoneda ${ }^{11}$, Atsushi Nakajima ${ }^{11}$, Tohru Funahashi ${ }^{6}$, Shigeru Miyazaki ${ }^{12}$, \\ Katsuto Tokunaga ${ }^{13}$, Hiroaki Masuzaki ${ }^{14}$, Takato Ueno ${ }^{15}$, Kazuyuki Hamaguchi ${ }^{16}$, Kiyoji Tanaka ${ }^{3}$, \\ Kentaro Yamada ${ }^{17}$, Toshiaki Hanafusa ${ }^{18}$, Shinichi Oikawa ${ }^{19}$, Hironobu Yoshimatsu ${ }^{20}$, Toshiie Sakata ${ }^{20}$, \\ Yuji Matsuzawa ${ }^{6}$, Kazuwa Nakao ${ }^{1,21}$ and Akihiro Sekine ${ }^{1,2}$
}

\begin{abstract}
Metabolic syndrome is defined as a cluster of multiple risk factors, including central obesity, dyslipidemia, hypertension and impaired glucose tolerance, that increase cardiovascular disease morbidity and mortality. Genetic factors are important in the development of metabolic syndrome, as are environmental factors. However, the genetic background of metabolic syndrome is not yet fully clarified. There is evidence that obesity and obesity-related phenotypes are associated with variations in several genes, including NEGR1, SEC16B, TMEM18, ETV5, GNPDA2, BDNF, MTCH2, SH2B1, FTO, MAF, MC4R, KCTD15, SCG3, MTMR9, TFAP2B, MSRA, LYPLAL1, GCKR and FADS1. To investigate the relationship between metabolic syndrome and variations in these genes in the Japanese population, we genotyped 33 single-nucleotide polymorphisms (SNPs) in 19 genes from 1096 patients with metabolic syndrome and 581 control individuals who had no risk factors for metabolic syndrome. Four SNPs in the FTO gene were significantly related to metabolic syndrome: rs9939609 $(P=0.00013)$, rs8050136 $(P=0.00011)$, rs1558902 $\left(P=6.6 \times 10^{-5}\right)$ and rs1421085 $\left(P=7.4 \times 10^{-5}\right)$. rs3764220 in the SCG3 gene $(P=0.0010)$ and rs2293855 in the MTMR9 gene $(P=0.0015)$ were also significantly associated with metabolic syndrome. SNPs in the FTO, SCG3 and MTMR9 genes had no SNP $\times$ SNP epistatic effects on metabolic syndrome. Our data suggest that genetic variations in the FTO, SCG3 and MTMR9 genes independently influence the risk of metabolic syndrome.
\end{abstract}

Journal of Human Genetics (2011) 56, 647-651; doi:10.1038/jhg.2011.74; published online 28 July 2011

Keywords: FTO; metabolic syndrome; MTMR9; SCG3

\section{INTRODUCTION}

Metabolic syndrome is a common clinical phenotype that is concurrent with metabolic abnormalities, including central obesity, glucose intolerance, dyslipidemia and hypertension. ${ }^{1}$ Several different definitions of the syndrome exist ${ }^{2}$ and there are still debates on the adequacy of the concept; however, metabolic syndrome has attracted considerable interest. Although the pathogenesis of metabolic syndrome is not fully understood, the predominant underlying risk factor is considered to be central obesity due to an atherogenic diet and physical inactivity in the presence of some genetic background., ${ }^{2,3}$ Adipose tissue, especially visceral fat, secretes various adipocytokines. An increase in adipose tissue mass leads to an alteration in the plasma levels of adipocytokines, resulting in the development of dyslipidemia, hypertension and insulin resistance. . $^{3,4}$

${ }^{1}$ EBM Research Center, Kyoto University Graduate School of Medicine, Kyoto, Japan; ${ }^{2}$ Center for Genomic Medicine, Unit of Genome Informatics, Kyoto University Graduate School of Medicine, Kyoto, Japan; ${ }^{3}$ Graduate School of Comprehensive Human Sciences, University of Tsukuba, Tsukuba, Japan; ${ }^{4}$ Health Science University, Yamanashi, Japan; ${ }^{5}$ Department of Hygiene, Faculty of Medicine, Kagawa University, Kagawa, Japan; ${ }^{6}$ Department of Metabolic Medicine, Graduate School of Medicine, Osaka University, Osaka, Japan; ${ }^{7}$ Rinku General Medical Center, Osaka, Japan; ${ }^{8}$ Toyonaka Municipal Hospital, Osaka, Japan; ${ }^{9}$ Otemae Hospital, Osaka, Japan; ${ }^{10}$ Department of Medicine and Clinical Science, Okayama University Graduate School of Medicine, Dentistry and Pharmaceutical Sciences, Okayama, Japan; ${ }^{11}$ Division of Gastroenterology, Yokohama City University Graduate School of Medicine, Yokohama, Japan; ${ }^{12}$ Tokyo Postal Services Agency Hospital, Tokyo, Japan; ${ }^{13}$ Itami City Hospital, Hyogo, Japan; ${ }^{14}$ Division of Endocrinology and Metabolism, Second Department of Internal Medicine, University of the Ryukyus Faculty of Medicine, Okinawa, Japan; ${ }^{15}$ Research Center for Innovative Cancer Therapy, Kurume University, Kurume, Japan; ${ }^{16}$ Department of Community Health and Gerontological Nursing, Faculty of Medicine, Oita University, Oita, Japan; ${ }^{17}$ Division of Endocrinology and Metabolism, Department of Medicine, Kurume University, Kurume, Japan; ${ }^{18}$ Department of Internal Medicine (I), Osaka Medical College, Osaka, Japan; ${ }^{19}$ Division of Endocrinology and Metabolism, Department of Medicine, Nippon Medical School, Tokyo, Japan; ${ }^{20}$ Department of Internal Medicine I, Faculty of Medicine, Oita University, Oita, Japan and ${ }^{21}$ Department of Medicine and Clinical Science, Kyoto University Graduate School of Medicine, Kyoto, Japan

Correspondence: Dr K Hotta, EBM Research Center, Kyoto University Graduate School of Medicine, Yoshida-Konoecho, Sakyo-ku, Kyoto 606-8501, Japan.

E-mail: kikukoh@kuhp.kyoto-u.ac.jp

Received 12 April 2011; revised 31 May 2011; accepted 8 June 2011; published online 28 July 2011 
Results from studies using twins and families have suggested that genetic and environmental factors contribute to the clustering of metabolic abnormalities in various ethnic groups. ${ }^{5-10}$ We determined in a previous study that four single-nucleotide polymorphisms (SNPs) (rs2294901, rs6133922, rs6077785 and rs6108572) in the McKusickKaufman syndrome $(M K K S)$ gene were significantly associated with metabolic syndrome in the Japanese population ${ }^{11}$ by screening SNPs in 85 obesity-related genes that had been reported as of $2005 . .^{12}$ We carried out a large-scale case-control association study and found that secretogranin III $(S C G 3)^{13}$ and myotubularin-related protein 9 $(\text { MTMR9 })^{14}$ conferred susceptibility to the obesity phenotype in the Japanese population. Recent genome-wide association studies revealed the SNPs associated with obesity and fat distribution (waist circumference and waist to hip ratio). ${ }^{15-19}$ We confirmed that some of these SNPs are also associated with obesity and visceral fat area as determined by computed tomography in the Japanese population. ${ }^{20-22}$

In this study, we investigated the association between metabolic syndrome and 33 SNPs related to obesity and the obesity-related phenotype. We found that SNPs in the fat mass and obesity associated (FTO), SCG3 and MTMR9 genes are associated with metabolic syndrome.

\section{MATERIALS AND METHODS}

\section{Study subjects}

The sample size of the group of Japanese subjects with metabolic syndrome was 1096 (male to female ratio, 594:502; age, $53.8 \pm 12.5$ years). The sample size of the group of Japanese controls was 581 (male to female ratio, 182:399; age, $47.2 \pm 14.8$ years). Metabolic syndrome was diagnosed as reported previously. ${ }^{11,23}$ In brief, metabolic syndrome is defined by the presence of two or more metabolic abnormalities in addition to obesity (body mass index $>25 \mathrm{~kg} \mathrm{~m}^{-2}$ ). The metabolic abnormalities were as follows: (1) triglyceride level $\geqslant 150 \mathrm{mg}$ per $100 \mathrm{ml}$ and/or high-density lipoprotein cholesterol level $<40 \mathrm{mg}$ per $100 \mathrm{ml}$, or under treatment for this type of dyslipidemia; (2) systolic blood pressure $\geqslant 130 \mathrm{~mm} \mathrm{Hg}$ and/or diastolic blood pressure blood pressure $\geqslant 85 \mathrm{~mm} \mathrm{Hg}$, or under treatment for hypertension; and (3) fasting glucose level $\geqslant 110 \mathrm{mg}$ per $100 \mathrm{ml}$, or under treatment for diabetes. The control group consisted of the subjects who were not obese (body mass index $<25 \mathrm{~kg} \mathrm{~m}^{-2}$ ) and who exhibited none of the metabolic abnormalities described above. Clinical characteristics of subjects are summarized in Table 1. Subjects with metabolic syndrome were recruited from outpatient clinics. Control subjects were selected from non-obese Japanese volunteers who had undergone a medical examination for common disease screening. Written informed consent was obtained from each subject and the protocol was approved by the ethics committee of each institution and by that of Kyoto University.

\section{DNA preparation and SNP genotyping}

Using Genomix (Talent Srl, Trieste, Italy), genomic DNA was extracted from blood samples collected from each subject. We constructed Invader probes (Third Wave Technologies, Madison, WI, USA) for rs3101336, rs2568958 and rs2815752 in the neuronal growth regulator 1 (NEGR1) gene; rs10913469 in the SEC16 homolog B (SEC16B) gene; rs6548238 and rs7561317 in the transmembrane protein 18 (TMEM18) gene; rs7647305 in the ets variant 5 (ETV5) gene; rs10938397 in the glucosamine-6-phosphate deaminase 2 (GNPDA2) gene; rs6265 and rs925946 in the brain-derived neurotrophic factor (BDNF) gene; rs10838738 in the mitochondrial carrier homolog 2 (MTCH2) gene; rs7498665 in the SH2B adaptor protein 1 (SH2B1) gene; rs9939609, rs8050136, rs6499640, rs1121980, rs1558902 and rs1421085 in the FTO gene; rs1424233 in the v-maf musculo-aponeurotic fibrosarcoma oncogene homolog (MAF) gene; rs17782313, rs12970134, rs489693 and rs17700144 in the melanocortin 4 receptor (MC4R) gene; rs29941 and rs11084753 in the potassium channel tetramerization domain-containing 15 (KCTD15) gene; rs3764220 in the SCG3 gene; rs2293855 in the MTMR9 gene; rs987237 in the transcription factor AP$2 \beta$ (TFAP2B) gene; rs7826222 in the methionine sulfoxide reductase A (MSRA) gene; rs2605100 in the lysophospholipase-like-1 (LYPLAL1) gene; rs780094 and
Table 1 Clinical characteristics of the subjects

\begin{tabular}{|c|c|c|c|}
\hline & Case & Control & P-value \\
\hline Gender (men/women) & $594 / 502$ & 182/399 & $<0.0001$ \\
\hline Age (years) & $53.8 \pm 12.5$ & $47.2 \pm 14.8$ & $<0.0001$ \\
\hline Body mass index $\left(\mathrm{kg} \mathrm{m}^{-2}\right)$ & $32.0 \pm 5.2$ & $21.4 \pm 2.1$ & $<0.0001$ \\
\hline Plasma glucose (mg per $100 \mathrm{ml})$ & $131.9 \pm 45.1$ & $90.9 \pm 7.7$ & $<0.0001$ \\
\hline Triglycerides (mg per $100 \mathrm{ml}$ ) & $184.0 \pm 145.3$ & $76.0 \pm 28.8$ & $<0.0001$ \\
\hline $\begin{array}{l}\text { High-density lipoprotein } \\
\text { cholesterol (mg per } 100 \mathrm{ml} \text { ) }\end{array}$ & $51.3 \pm 18.0$ & $67.3 \pm 14.0$ & $<0.0001$ \\
\hline Systolic blood pressure $(\mathrm{mm} \mathrm{Hg})$ & $139.9 \pm 17.0$ & $111.1 \pm 10.2$ & $<0.0001$ \\
\hline Diastolic blood pressure $(\mathrm{mm} \mathrm{Hg})$ & $85.3 \pm 12.2$ & $69.4 \pm 7.5$ & $<0.0001$ \\
\hline
\end{tabular}

Prevalence of metabolic disease Dyslipidemia

Hypertension

Impaired fasting glucose

$858(78 \%)$

$974(89 \%)$

0

$770(70 \%)$

No. of subjects under treatment

Dyslipidemia

Hypertension

319

466

Diabetes

379

Data are shown as the mean \pm s.d. Men/women ratio was analyzed by the $z^{2}$-test. $P$-values for quantitative traits were analyzed using the Mann-Whitney U-test.

rs1260326 in the glucokinase regulator (GCKR) gene; and rs174547 in the fatty acid desaturase 1 (FADS1) gene. The SNPs were genotyped using Invader assays as previously described. ${ }^{24}$ The success rate of these assays was $>99.0 \%$.

\section{Statistical analysis}

For the additive model, we coded genotypes as 0,1 or 2, depending on the number of copies of the risk alleles. For the dominant model, homozygosity and heterozygosity with the risk allele were coded as 1 and the other was coded as 0 . For the recessive model, homozygosity with the risk allele was coded as 1 and others were coded as 0 . Additive, dominant and recessive models were chosen for each SNP, according to data from the previous reports. ${ }^{13-19}$ Odds ratios (ORs) and $P$-values adjusted for age and gender were calculated using multiple logistic regression analysis with genotypes, age and gender as the independent variables. The Hardy-Weinberg equilibrium was assessed using the $\chi^{2}$-test. ${ }^{25}$ Simple comparison of the clinical data between case and control groups was carried out using the Mann-Whitney $U$-test. To test $\mathrm{SNP} \times \mathrm{SNP}$ epistasis for case-control population-based samples, we used the logistic regression model for each SNP1 and SNP2, and fits the model in the form of $Y=\beta_{0}+\beta_{1} \times \mathrm{SNP} 1+\beta_{2} \times \mathrm{SNP} 2+\beta_{3} \times \mathrm{SNP} 1 \times \mathrm{SNP} 2+\beta_{4} \times$ age $+\beta_{5} \times$ gender. Statistical analysis was performed using the software R (http://www.r-project. org/). $P$-values were corrected by Bonferroni adjustment and $P<0.00152(0.05 /$ 33) was considered significant.

We examined the power $(\alpha=0.00152)$ of the test for 1096 cases and 581 controls using GDesignPlus (StaGen, Tokyo, Japan). We considered the controls of five different levels of minor allele frequencies (0.1-0.5) and four different levels of the ORs (1.2-1.5). Obtained power was indicated in Supplementary Table 1.

\section{RESULTS}

We used measurements of body mass index $\left(>25 \mathrm{~kg} \mathrm{~m}^{-2}\right)$ instead of waist circumference, as there are still debates on the criteria for waist circumference, especially in Japanese women. The prevalence of metabolic syndrome increased with subject age and has been previously reported to be $\sim 6-7$ times higher in men than in women in Japan. ${ }^{23}$ Among our subjects, the ratio of men to women and the average age were both significantly higher in the case group than in the control group (Table 1). To adjust for the effects of age and gender, logistic regression analysis was performed. The most significant 
Table 2 Genotype and association tests

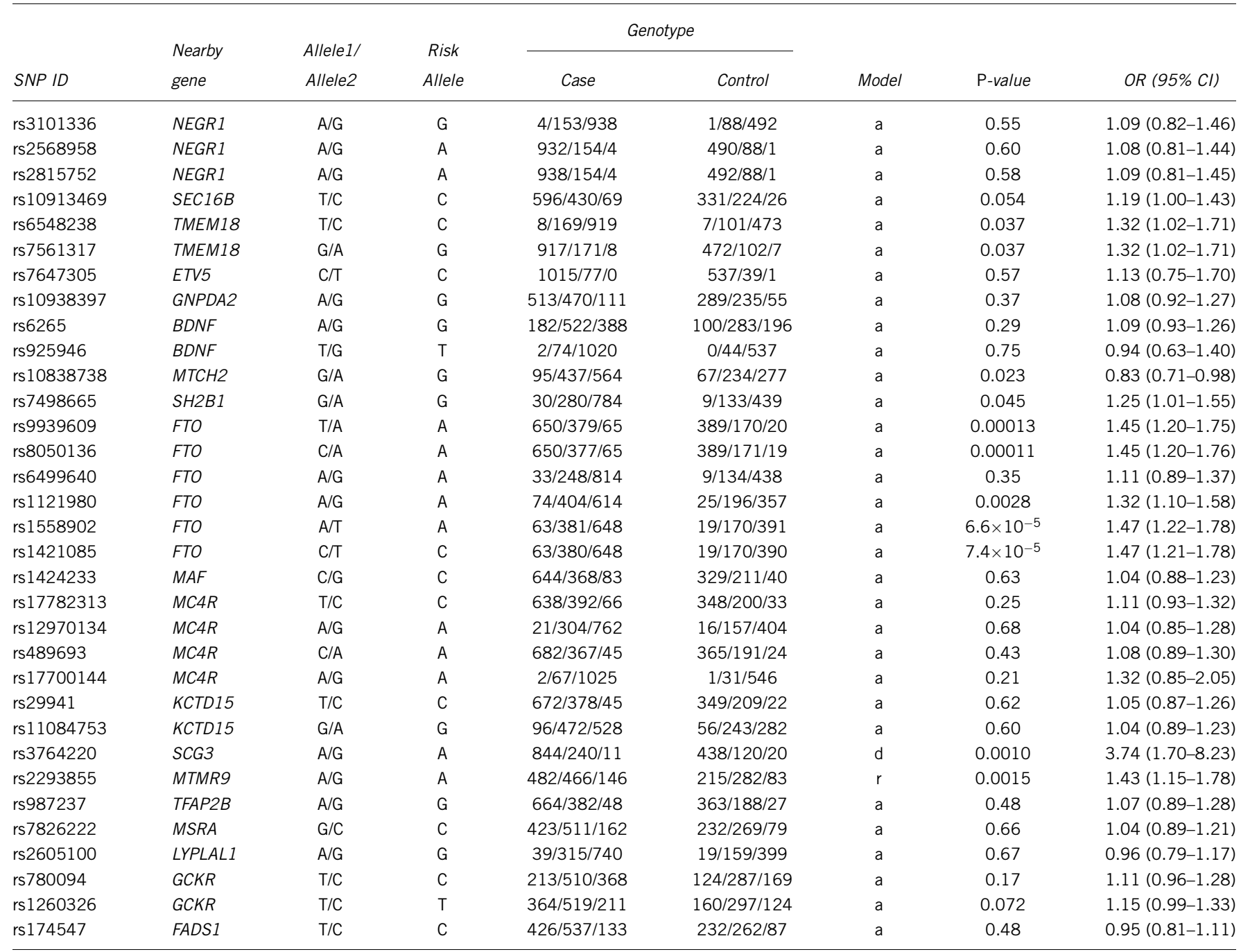

Abbreviations: a, additive model; $\mathrm{Cl}$, confidence interval; $d$, dominant model; OR, odds ratio; $r$, recessive model; SNP, single-nucleotide polymorphism.

The OR for each SNP was adjusted simultaneously for age and gender.

associations were observed for $\mathrm{rs} 1558902\left(P=6.6 \times 10^{-5}\right.$, allele-specific OR (95\% confidence interval $(\mathrm{CI})$ adjusted for age and gender $)=1.47$ $(1.22-1.78))$ and $\mathrm{rs} 1421085\left(P=7.4 \times 10^{-5}\right.$, allele-specific OR $(95 \% \mathrm{CI}$ adjusted for age and gender $)=1.47(1.21-1.77))$ in the FTO gene (Table 2). These two SNPs were previously reported as variations associated with waist circumference ${ }^{18,19}$ and associated with visceral fat area as determined by computed tomography. ${ }^{22}$ Two other SNPs (rs9939609 and rs8050136) in the FTO gene were also significantly associated with metabolic syndrome even when the conservative Bonferroni's correction was applied $(P<0.00152)$. Significant associations were also observed between metabolic syndrome and rs3764220 in the SCG3 gene $(P=0.0010)$ and rs2293855 in the MTMR9 gene $(P=0.00146)$. SNPs, rs6548238 and rs7561317 in the TMEM18 gene, rs7498665 in the SH2B1 gene and rs1121980 in the FTO gene, were marginally associated with metabolic syndrome $(P<0.05)$. SNP rs 10838738 in the $M T C H 2$ gene was also marginally associated with metabolic syndrome, although presence of the G-allele, which is a risk allele for obesity, ${ }^{15,16}$ was associated with a reduced risk of metabolic syndrome. Other SNPs did not show any significant associations with metabolic syndrome. The lack of significant association of these SNPs is most likely due to the relatively lower power of this study
Table 3 Results for SNP pairs for epistasis in case-control analysis

\begin{tabular}{lccc}
\hline SNP1 & SNP2 & OR $(95 \% \mathrm{Cl})$ & Epistasis P-value \\
\hline rs2293855 & rs3764220 & $0.07(0.01-0.70)$ & 0.023 \\
rs2293855 & rs1421085 & $0.80(0.55-1.18)$ & 0.27 \\
rs2293855 & rs1558902 & $0.81(0.55-1.19)$ & 0.29 \\
rs2293855 & rs8050136 & $0.84(0.57-1.24)$ & 0.38 \\
rs2293855 & rs9939609 & $0.82(0.56-1.21)$ & 0.32 \\
rs3764220 & rs1421085 & $1.60(0.44-5.82)$ & 0.48 \\
rs3764220 & rs1558902 & $1.60(0.44-5.84)$ & 0.47 \\
rs3764220 & rs8050136 & $1.58(0.43-5.74)$ & 0.49 \\
rs3764220 & rs9939609 & $1.57(0.43-5.71)$ & 0.49 \\
\hline
\end{tabular}

Abbreviations: $\mathrm{Cl}$, confidence interval; OR, odds ratio; SNP, single-nucleotide polymorphism.

(Supplementary Table 1). All SNPs were in Hardy-Weinberg equilibrium $(P>0.05)$, with the exception of rs6499640 $(P=0.0099)$ and rs1424233 $(P=0.0034)$ in the case group and rs3764220 in the control subjects $(P=0.0018)$.

As six SNPs were significantly associated with metabolic syndrome, we tested $\mathrm{SNP} \times \mathrm{SNP}$ epistasis. Four SNPs in the FTO gene were in a 
Table 4 Genotype and association tests of dyslipidemia, hypertension and impaired fasting glucose

\begin{tabular}{|c|c|c|c|c|c|c|c|c|c|c|}
\hline & \multirow{2}{*}{$\begin{array}{l}\text { Control } \\
\text { Genotype }\end{array}$} & \multicolumn{3}{|c|}{ Dyslipidemia $(n=858)$} & \multicolumn{3}{|c|}{ Hypertension $(\mathrm{n}=974)$} & \multicolumn{3}{|c|}{ Impaired fasting glucose $(\mathrm{n}=770)$} \\
\hline & & Genotype & P-value & OR $(95 \% \mathrm{Cl})$ & Genotype & P-value & OR $(95 \% \mathrm{Cl})$ & Genotype & P-value & OR $(95 \% \mathrm{Cl})$ \\
\hline rs8050136 & $389 / 171 / 19$ & $511 / 295 / 48$ & 0.00045 & $1.43(1.17-1.74)$ & $575 / 339 / 57$ & $8.9 \times 10^{-5}$ & $1.48(1.22-1.80)$ & $458 / 265 / 44$ & 0.00020 & $1.47(1.20-1.80)$ \\
\hline rs1558902 & 19/170/391 & $47 / 298 / 510$ & 0.00024 & $1.45(1.19-1.77)$ & $55 / 344 / 573$ & $4.8 \times 10^{-5}$ & $1.50(1.23-1.83)$ & $43 / 268 / 456$ & 0.00011 & $1.49(1.22-1.83)$ \\
\hline rs1421085 & 19/170/390 & 47/297/509 & 0.00026 & $1.45(1.19-1.77)$ & $55 / 343 / 573$ & $5.4 \times 10^{-5}$ & $1.50(1.23-1.82)$ & $43 / 267 / 456$ & 0.00012 & $1.49(1.22-1.83)$ \\
\hline
\end{tabular}

Abbreviations: $\mathrm{Cl}$, confidence interval; OR, odds ratio.

The OR for each single-nucleotide polymorphism was adjusted simultaneously for age and gender.

linkage disequilibrium $\left(r^{2}>0.97\right)$, thus, pair of these SNPs were not analyzed. Any pairs of SNPs did not show significant epistatic effect on metabolic syndrome (Table 3). We performed the multiple logistic regression analysis with three genotypes (rs1558902, rs3764220 and rs2293855), age and gender as the independent variables and found that effects of these SNPs in the three genes on metabolic syndrome were additive: rs 1558902 (additive model), $P=4.1 \times 10^{-5}$, OR $(95 \%$ $\mathrm{CI})=1.50$ (1.23-1.81); rs3764220 (dominant model), $P=0.00018$, OR

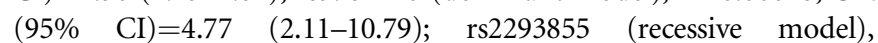
$P=0.00090$, OR $(95 \% \mathrm{CI})=1.46(1.17-1.82)$. Similar results were obtained using rs9939609, rs8050136 and rs1421085, instead of rs1558902.

Next, we examined the effects of significant SNPs on each metabolic disorder (dyslipidemia, hypertension and impaired fasting glucose). SNPs (rs9939609, rs8050136, rs1558902 and rs1421085) in the FTO gene were significantly associated with dyslipidemia, hypertension and impaired fasting glucose (Table 4). rs2293855 in the MTMR9 gene was significantly associated with hypertension and impaired fasting glucose, and was marginally associated with dyslipidemia. rs3764220 in the SCG3 gene was marginally associated with all three metabolic disorders. There were no obvious differences among the effects (OR) on each metabolic disorder.

\section{DISCUSSION}

The SNPs that were most significantly associated with metabolic syndrome (rs1558902 and rs1421085) exist in the FTO gene. Visceral fat accumulation is the most predominant factor for the development of metabolic syndrome. SNPs (rs1558902 and rs1421085) were reported to be associated with waist circumference and visceral fat area as measured by computed tomography. ${ }^{18,19,22}$ SNPs (rs9939609, rs8050136, rs1558902 and rs1421085) were also associated with obesity and type 2 diabetes. ${ }^{15-17}$ We have previously reported that rs3764220 in the SCG3 gene was associated with obesity and subcutaneous fat area as measured by computed tomography ${ }^{13}$ and that rs2293855 in the MTMR9 gene was associated with obesity and hypertension. $^{14}$ Therefore, it is likely that rs9939609, rs8050136, rs1558902 and rs1421085 in the FTO, rs3764220 in the SCG3 and rs2293855 in the MTMR9 genes would be susceptible for metabolic syndrome. Epistasis, or gene-gene interaction, has recently received much attention in human genetics. ${ }^{26}$ In this study, effect of these SNPs on metabolic syndrome was independent, and epistatic effect was not observed.

In the simulation study, the power of this test was 0.317 in the following condition; the sizes of the case and the control groups are 1096 and 581, respectively, OR is 1.4 , risk allele frequency is 0.2 and the model of inheritance is additive. Therefore, further studies would be necessary to elucidate the association between SNPs and metabolic syndrome.

SNPs (rs780094 and rs1260326) in the GCKR gene were previously reported to be associated with metabolic disorders; ${ }^{27-29}$ however, the present study did not show the association with metabolic syndrome. The C-allele of rs780094 was reported to be associated with increased fasting plasma glucose and lower triglyceride levels. The risk allele of rs780094 had an antagonistic affect on plasma glucose and triglycerides levels. SNP rs174547 in the FADS1 gene, which is in almost complete linkage disequilibrium with rs174550, is associated with dyslipidemia and type 2 diabetes. ${ }^{28,30}$ Similar to rs780094, the effects of the risk allele of rs174547 on type 2 diabetes, hypertension and dyslipidemia are not the same. Thus, rs780094 and rs174550 are not likely to be important in the development of metabolic syndrome.

SNPs (rs6548238 and rs7561317) in the TMEM18 gene were marginally associated with metabolic syndrome. We have reported that these SNPs are associated with obesity. ${ }^{21}$ Takeuchi et al. reported that rs48454344, which is in almost complete linkage disequilibrium with rs6548238 and rs7561317, ${ }^{21}$ was associated with body mass index and type 2 diabetes. $^{31}$ Therefore, rs6548238 and rs7561317 in the TMEM18 gene might be susceptible for the development of metabolic syndrome.

The G-allele of rs10838738 in the MTCH2 gene, which is a risk allele for obesity, ${ }^{16}$ reduced the risk of metabolic syndrome. Takeuchi et al. ${ }^{31}$ reported that the G-allele is a risk factor for obesity and is protective for type 2 diabetes. Although further investigations would be necessary, there is a possibility that there are some variations that are specifically associated with components of metabolic syndrome, apart from obesity.

Our approach effectively indicated that SNPs in the FTO, SCG3 and MTMR9 genes are associated with metabolic syndrome in the Japanese population, in addition to the previously reported SNPs in the MKKS gene. SNPs in the FTO, SCG3 and MTMR9 genes had no SNP $\times$ SNP epistatic effects on metabolic syndrome. As metabolic syndrome is a complex concept and the criteria are still controversial, further investigations are necessary to elucidate the roles of SNPs in the MKKS, FTO, SCG3 and MTMR9 genes in the development of metabolic syndrome.

\section{CONFLICT OF INTEREST}

The authors declare no conflict of interest.

\section{ACKNOWLEDGEMENTS}

This work was supported by a Grant-in-Aid from the Ministry of Education, Science, Sports, and Culture of Japan (21591186) and by the Mitsui Life Science Social Welfare Foundation. 
1 Carr, D. B., Utzschneider, K. M., Hull, R. L., Kodama, K., Retzlaff, B. M., Brunzell, J. D. et al. Intra-abdominal fat is a major determinant of the National Cholesterol Education Program Adult Treatment Panel III criteria for the metabolic syndrome. Diabetes 53, 2087-2094 (2004).

2 Pollex, R. L. \& Hegele, R. A. Genetic determinants of the metabolic syndrome. Nat. Clin. Pract. Cardiovasc. Med. 3, 482-489 (2006).

3 Matsuzawa, Y. Therapy insight: adipocytokines in metabolic syndrome and related cardiovascular disease. Nat. Clin. Pract. Cardiovasc. Med. 3, 35-42 (2006).

4 Hotta, K., Funahashi, T., Bodkin, N. L., Ortmeyer, H. K., Arita, Y., Hansen, B. C. et al. Circulating concentrations of the adipocyte protein adiponectin are decreased in parallel with reduced insulin sensitivity during the progression to type 2 diabetes in rhesus monkeys. Diabetes 50, 1126-1133 (2001).

5 Carmelli, D., Cardon, L. R. \& Fabsitz, R. Clustering of hypertension, diabetes, and obesity in adult male twins: same genes or same environments? Am. J. Hum. Genet. 55, 566-573 (1994).

6 Edwards, K. L., Newman, B., Mayer, E., Selby, J. V., Krauss, R. M. \& Austin, M. A Heritability of factors of the insulin resistance syndrome in women twins. Genet. Epidemiol. 14, 241-253 (1997).

7 Austin, M. A., Edwards, K. L., McNeely, M. J., Chandler, W. L., Leonetti, D. L., Talmud, P. J. et al. Heritability of multivariate factors of the metabolic syndrome in nondiabetic Japanese Americans. Diabetes 53, 1166-1169 (2004).

8 Chen, W., Srinivasan, S. R., Elkasabany, A. \& Berenson, G. S. The association of cardiovascular risk factor clustering related to insulin resistance syndrome (Syndrome $\mathrm{X}$ ) between young parents and their offspring: the Bogalusa Heart Study. Atherosclerosis 145, 197-205 (1999).

9 Lee, K. E., Klein, B. E. \& Klein, R. Familial aggregation of components of the multiple metabolic syndrome in the Framingham Heart and Offspring Cohorts: genetic analysis workshop problem 1. BMC Genet. 4, S94-S98 (2003).

10 Wu, K. D., Hsiao, C. F., Ho, L. T., Sheu, W. H., Pei, D., Chuang, L. M. et al. Clustering and heritability of insulin resistance in Chinese and Japanese hypertensive families: a Stanford-Asian Pacific Program in Hypertension and Insulin Resistance Sibling Study. Hypertens. Res. 25, 529-536 (2002)

11 Hotta, K. Nakamura, T., Takasaki, J., Takahashi, H., Takahashi, A., Nakata, Y et al. Screening of 336 single-nucleotide polymorphisms in 85 obesity-related genes revealed McKusick-Kaufman syndrome gene variants are associated with metabolic syndrome. J. Hum. Genet. 54, 230-235 (2009).

12 Rankinen, T., Zuberi, A., Chagnon, Y. C., Weisnagel, S. J., Argyropoulos, G., Walts, B. et al. The human obesity gene map: the 2005 update. Obesity 14, 529-644 (2006).

13 Tanabe, A., Yanagiya, T., Iida, A., Saito, S., Sekine, A., Takahashi, A. et al. Functional single-nucleotide polymorphisms in the secretogranin III (SCG3) gene that form secretory granules with appetite-related neuropeptides are associated with obesity. J. Clin. Endocrinol. Metab. 92, 1145-1154 (2007).

14 Yanagiya, T., Tanabe, A., Iida, A., Saito, S., Sekine, A., Takahashi, A. et al. Association of single-nucleotide polymorphisms in MTMR9 gene with obesity. Hum. Mol. Genet. 16, 3017-3026 (2007).

15 Thorleifsson, G., Walters, G. B., Gudbjartsson, D. F., Steinthorsdottir, V., Sulem, P., Helgadottir, A. et al. Genome-wide association yields new sequence variants at seven loci that associate with measures of obesity. Nat. Genet. 41, 18-24 (2009).
16 Willer, C. J., Speliotes, E. K., Loos, R. J., Li, S., Lindgren, C. M., Heid, I. M. et al. Six new loci associated with body mass index highlight a neuronal influence on body weight regulation. Nat. Genet. 41, 25-34 (2009).

17 Meyre, D., Delplanque, J., Chèvre, J. C., Lecoeur, C., Lobbens, S., Gallina, S. et al. Genome-wide association study for early-onset and morbid adult obesity identifies three new risk loci in European populations. Nat. Genet. 41, 157-159 (2009).

18 Lindgren, C. M., Heid, I. M., Randall, J. C., Lamina, C., Steinthorsdottir, V., Qi, L. et al. Genome-wide association scan meta-analysis identifies three loci influencing adiposity and fat distribution. PLoS Genet. 5, e1000508 (2009).

19 Heard-Costa, N. L., Zillikens, M. C., Monda, K. L., Johansson, A., Harris, T. B., Fu, M. et al. NRXN3 is a novel locus for waist circumference: a genome-wide association study from the CHARGE consortium. PLoS Genet. 5, e1000539 (2009).

20 Hotta, K., Nakata, Y., Matsuo, T., Kamohara, S., Kotani, K., Komatsu, R. et al. Variations in the FTO gene are associated with severe obesity in the Japanese. J. Hum. Genet. 53, 546-553 (2008).

21 Hotta, K., Nakamura, M., Nakamura, T., Matsuo, T., Nakata, Y., Kamohara, S. et al. Association between obesity and polymorphisms in SEC16B, TMEM18, GNPDA2, BDNF, FAIM2 and MC4R in a Japanese population. J. Hum. Genet. 54, 727-731 (2009).

22 Hotta, K., Nakamura, M., Nakamura, T., Matsuo, T., Nakata, Y., Kamohara, S. et al. Polymorphisms in NRXN3, TFAP2B, MSRA, LYPLAL1, FTO and MC4R and their effect on visceral fat area in the Japanese population. J. Hum. Genet. 55, 738-742 (2010).

23 Arai, H., Yamamoto, A., Matsuzawa, Y., Saito, Y., Yamada, N., Oikawa, S. et al. Prevalence of metabolic syndrome in the general Japanese population in 2000. J. Atheroscler. Thromb. 13, 202-208 (2006).

24 Ohnishi, Y., Tanaka, T., Ozaki, K., Yamada, R., Suzuki, H. \& Nakamura, Y. A highthroughput SNP typing system for genome-wide association studies. J. Hum. Genet. 46, 471-477 (2001).

25 Nielsen, D. M., Ehm, M. G. \& Weir, B. S. Detecting marker-disease association by testing for Hardy-Weinberg disequilibrium at a marker locus. Am. J. Hum. Genet. 63, 1531-1540 (1998).

26 Cordell, H. J. Detecting gene-gene interactions that underlie human diseases. Nat. Rev. Genet. 10, 392-404 (2009).

27 Bi, M., Kao, W. H., Boerwinkle, E., Hoogeveen, R. C., Rasmussen-Torvik, L. J., Astor, B. C. et al. Association of rs 780094 in GCKR with metabolic traits and incident diabetes and cardiovascular disease: the ARIC Study. PLoS One 5, e11690 (2010).

28 Dupuis, J., Langenberg, C., Prokopenko, I., Saxena, R., Soranzo, N., Jackson, A. U. et al. New genetic loci implicated in fasting glucose homeostasis and their impact on type 2 diabetes risk. Nat. Genet. 42, 105-116 (2010).

29 Onuma, H., Tabara, Y., Kawamoto, R., Shimizu, I., Kawamura, R., Takata, Y. et al. The GCKR rs780094 polymorphism is associated with susceptibility of type 2 diabetes, reduced fasting plasma glucose levels, increased triglycerides levels and lower HOMAIR in Japanese population. J. Hum. Genet. 55, 600-604 (2010).

30 Kathiresan, S., Willer, C. J., Peloso, G. M., Demissie, S., Musunuru, K., Schadt, E. E. et al. Common variants at 30 loci contribute to polygenic dyslipidemia. Nat. Genet. 41, 56-65 (2009).

31 Takeuchi, F., Yamamoto, K., Katsuya, T., Nabika, T., Sugiyama, T., Fujioka, A. et al. Association of genetic variants for susceptibility to obesity with type 2 diabetes in Japanese individuals. Diabetologia 54, 1350-1359 (2011).

Supplementary Information accompanies the paper on Journal of Human Genetics website (http://www.nature.com/jhg) 